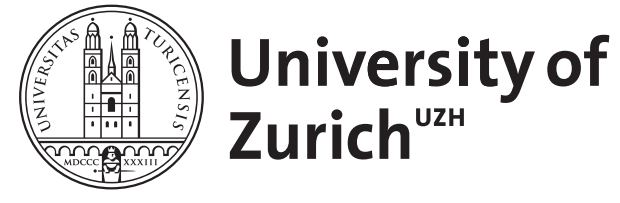

\title{
Velocity echoes in water
}

\author{
Hamm, Peter
}

\begin{abstract}
A three-point velocity correlation function $\mathrm{v}(\mathrm{t}(1)+\mathrm{t}(2)) \mathrm{v} 2(\mathrm{t}(1)) \mathrm{v}(0)$ is introduced for a better understanding of the recent 2D-Raman- $\mathrm{THz}$ spectroscopy of the intermolecular degrees of freedoms of water and aqueous salt solutions. This correlation function reveals echoes in the presence of inhomogeneous broadening, which are coined velocity echoes. In analogy to the well-known two-point velocity correlation function $\mathrm{v}(\mathrm{t}) \mathrm{v}(0)$, it reflects the density of states (DOS) of the system under study without having to amend them with transition dipoles and transition polarizabilities. The correlation function can be calculated from equilibrium trajectories and converges extremely quickly. After deriving the theory, the information content of the three-point velocity correlation function is first tested based on a simple harmonic oscillator model with Langevin dynamics. Subsequently, velocity echoes of TIP4P/2005 water are calculated as a function of temperature, covering ambient conditions, the supercooled regime and amorphous ice, as well as upon addition of various salts. The experimentally observed trends can be reproduced qualitatively with the help of computationally very inexpensive molecular dynamics simulations.
\end{abstract}

DOI: https://doi.org/10.1063/1.5112163

Posted at the Zurich Open Repository and Archive, University of Zurich

ZORA URL: https://doi.org/10.5167/uzh-183163

Journal Article

Accepted Version

Originally published at:

Hamm, Peter (2019). Velocity echoes in water. Journal of Chemical Physics, 151(5):054505.

DOI: https://doi.org/10.1063/1.5112163 


\title{
Velocity Echoes in Water
}

\author{
Peter Hamm \\ Department of Chemistry, University of Zurich, \\ Winterthurerstr. 190, CH-8057 Zürich, Switzerland \\ peter.hamm@chem.uzh.ch
}

(Dated: July 19, 2019)

\begin{abstract}
A three-point velocity correlation function $\left\langle v\left(t_{1}+t_{2}\right) v^{2}\left(t_{1}\right) v(0)\right\rangle$ is introduced for a better understanding of the recent 2D-Raman- $\mathrm{THz}$ spectroscopy of the intermolecular degrees of freedoms of water and aqueous salt solutions. That correlation function reveals echoes in the presence of inhomogeneous broadening, which are coined "velocity echoes". In analogy to the well-known twopoint velocity correlation function $\langle v(t) v(0)\rangle$, it reflects the density of states of the system under study without having to amend them with transition dipoles and transition polarizabilities. The correlation function can be calculate from equilibrium trajectories and converges extremely quickly. After deriving the theory, the information content the three-point velocity correlation function is first tested based on a simple harmonic oscillator model with Langevin dynamics. Subsequently, velocity echoes of TIP4P/2005 water are calculated as a function of temperature, covering ambient conditions, the super-cooled regime and amorphous ice, as well as upon addition of various salts. The experimentally observed trends can be reproduced qualitatively with the help of computationally very inexpensive molecular dynamics simulations.
\end{abstract}

Water obviously is a very important, and at the same time very fascinating liquid with many anomalies in its thermodynamical properties, ${ }^{1}$ culminating in the hypothesized liquid-to-liquid phase transition in its deeply supercooled regime. ${ }^{2,3}$ It is the directionality of the hydrogen-bonding between water molecules, forming networks of connected water molecules, that is responsible for the peculiar properties of water. With the introduction of hybrid 2D-Raman-THz techniques, ${ }^{4-7}$ it became possible recently to measure the $2 \mathrm{D}$ response of water in a very low frequency regime, where these intermolecular hydrogen-bond modes are found. 2D spectroscopic methods can enhance the information that can be extracted from the very blurred spectra in this spectral range, and indeed, echoes have been observed. In such a multi-pulse experiment, a first laser pulse impulsively excited all modes of the system, which however dephase very quickly due to homogeneous and/or inhomogeneous broadening. A second laser pulse "inverts the coherence", and the part of dephasing that is reversible, i.e., the inhomogeneous contribution, can rephase and form an echo at a time $t_{2}$ after the second pulse, that equals the time separation $t_{1}$ between the two laser pulses. The size and lifetime of an echo reflects the inhomogeneous broadening of the absorption spectra.

It is important to understand the difference between echoes from isolated probe molecules in a heterogeneous environment, which is the situation common for $\mathrm{NMR},{ }^{8}$ vibrational $^{9,10}$ or electronic spectroscopy ${ }^{11,12}$ vs echoes in 2D-Raman- $\mathrm{THz}$ spectroscopy. In the latter case, one is looking at the low-frequency, inter-molecular modes of a sample with an irregular structure, such as a liquid, a glass or a protein. Any snapshot structure of such a sample will reveal a wide distribution of modes, and hence should reveal an echo. The crucial question then is the timescale of the interconversion between structures that will determine whether the set of modes during the first and the second time periods $t_{1}$ and $t_{2}$, respectively, are the same. The lifetime of an echo hence reflects the lifetime of local structures on a length-scale that equals the delocalization length of the intermolecular modes.

In our experiments, the echoes became more pronounced when adding salts to the solution to artificially increase the inhomogeneity of the hydrogen-bond networks, ${ }^{6}$ when cooling neat water that makes those networks more persistent, ${ }^{7}$ or when deuterating water that makes hydrogen bonds slightly stronger. ${ }^{7}$ We do consider these echoes, and their dependence on external parameters, the most important outcome from 2D-Raman- $\mathrm{THz}$ spectroscopy so far. Recently, we fitted the 2D-Raman$\mathrm{THz}$ response to a model consisting of an ensemble of anharmonic oscillators, augmented with electrical anharmonicity, suggesting that the low frequency bands are indeed in the "quasi-inhomogenous" limit. ${ }^{13}$

However, attempts to reproduce these echoes from atomistic molecular dynamics (MD) simulations have not been overly successful. ${ }^{14}$ The reason is two-fold: First, the corresponding three-point correlation functions (see Eq. 8 below) converge extremely slowly and require simulation times of many $100 \mu \mathrm{s}$ (albeit for very small simulation boxes). These long simulation times can be dealt with using empirical water force fields, but are prohibitive for more sophisticated and presumably more accurate force fields such as MB-pol. ${ }^{15}$ Convergence of these correlation functions is so expensive since their calculation includes the "stability matrix" (even though methods have been proposed that circumvent its explicit calculation $^{16}$ ) and one faces the chaotic properties of classical mechanics systems. Second, it has been shown that the 2D-Raman- $\mathrm{THz}$ response depends extremely critically on the level of description of polarizability in a water force field. ${ }^{14,17}$ Contributions with different signs add up to the overall signal (e.g. electrical vs mechanical anharmonicity), ${ }^{13}$ and the net signal is the outcome of 
strong cancellation effects. Consequently, small changes to one parameter can completely change the overall response.

Here, a very easy to calculate three-point velocity correlation function $\left\langle v\left(t_{1}+t_{2}\right) v^{2}\left(t_{1}\right) v(0)\right\rangle$ is proposed as a way to circumvent both these problems. First, it converges extremely quickly, requiring only a few nanoseconds rather than 100's of $\mu \mathrm{s}$ of simulation time. Second, in analogy to the well known two-point velocity autocorrelation function $\langle v(t) v(0)\rangle$, it reflects the density of states (DOS) without having to amend them with proper transition dipoles or transition polarizabilities. This particular velocity correlation function does reveal echoes in the presence of inhomogeneous broadening; they are coined "velocity echoes". Despite the fact that this threepoint velocity correlation function will not allow one to directly fit an experimental 2D-Raman- $\mathrm{THz}$ spectrum, it still reveals to what extent a particular microscopic water force field results in inhomogeneously broadened bands in the frequency range of the intermolecular modes.

The approach presented here is conceptually similar to temperature quench echoes introduced quite some time ago. ${ }^{18-20}$ In these numerical experiments, the MD simulation of either a Lennard-Jones glass ${ }^{18}$ or a protein ${ }^{19,20}$ has been perturbed twice by setting all velocities to zero, each time (roughly) halving the temperature. Subsequently, a small but very sharp-in-time transient drop of temperature has been observed at a time after the second perturbation, which equaled the time between the two perturbations. As a generalization of this concept, "velocity reassignment echoes" have been introduced as well, ${ }^{21,22}$ which avoid the caveat that the temperature is halved with each perturbation. This works introduced the notion that echoes can also occur in purely classical mechanics systems. The "inversion of coherence" does not require quantum-mechanics (which is a common misconception); all it requires are close-to-harmonic modes that are not over-damped.

The simulations used to produce temperature quench echoes or velocity reassignment echoes involve nonequilibrium trajectories, and the perturbations need to be quite extreme (e.g., zeroing or inverting all velocities) in order to see an effect with affordable computational cost. In the limit of a small perturbation of the velocities, the temperature quench echo would probably converge to a response function that is closely related to the velocity echo introduced here. Yet, the velocity echo can be calculated from an equilibrium trajectory without any perturbation, and consequently converges much more quickly, as we will see.

\section{THEORY}

\section{A. Linear Response Functions}

To set the stage, the (1D) $\mathrm{THz}$ or Raman spectrum of a system is introduced, which is related to the following linear response functions, respectively:

$$
\begin{aligned}
& R_{1}^{(\mathrm{T})}(t)=-\frac{i}{\hbar} \operatorname{Tr}\left(\hat{\mu}(t)\left[\hat{\mu}(0), \hat{\rho}_{\mathrm{eq}}\right]\right) \\
& R_{1}^{(\mathrm{R})}(t)=-\frac{i}{\hbar} \operatorname{Tr}\left(\hat{\Pi}(t)\left[\hat{\Pi}(0), \hat{\rho}_{\mathrm{eq}}\right]\right) .
\end{aligned}
$$

Here, $[. ., .$.$] is a commutator, \hat{\mu}(t)$ the dipole operator at time $t, \hat{\Pi}(t)$ the polarisibility operator, and $\hat{\rho}_{\text {eq }}$ the equilibrium density matrix. In the most simple approximation, neglecting electrical anharmonicity, one can write:

$$
\begin{aligned}
\hat{\mu}(t) & \propto \hat{q}(t)+\ldots \\
\hat{\Pi}(t) & \propto \hat{q}(t)+\ldots
\end{aligned}
$$

where $\hat{q}(t)$ is the position operator of the involved modes, and the proportionality factors are the transition dipole and the transition polarizability, respectively. Hence, linear response can be recast in a generic form:

$$
R_{1}(t)=-\frac{i}{\hbar} \operatorname{Tr}\left(\hat{q}(t)\left[\hat{q}(0), \hat{\rho}_{\mathrm{eq}}\right]\right)
$$

In the classical limit, which should be a reasonable approximation for low-frequency intermolecular modes with $\hbar \omega \lesssim k_{B} T$, the commutator is replaced by a Poisson bracket, $1 / i \hbar[. ., ..] \rightarrow\{. . . .$.$\} , and one obtains (see Ap-$ pendix A 1 for a derivation): ${ }^{23}$

$$
R_{1}(t)=-\frac{1}{k_{B} T}\langle q(t) \dot{q}(0)\rangle,
$$

where the average $\langle\ldots\rangle$ is over molecular configurations along a long enough equilibrium trajectory, and over all degrees of freedom of the system, i.e., over the many molecules in a molecular dynamics (MD) simulation box as well as their $(x, y, z)$-coordinates.

The linear spectrum is related to the Fourier transformation of the time-derivative of the response function:

$$
I_{1}(\omega)=\frac{1}{k_{B} T} \Re \int_{0}^{\infty}\langle v(t) v(0)\rangle e^{i \omega t} d t
$$

with $v(t)=\dot{q}(t)$. The central property is the two-point velocity correlation function:

$$
c_{1}(t)=\langle v(t) v(0)\rangle,
$$

which converges very quickly, since correlation times are typically short, and since a single MD simulation box already contains $3 N$ samples (where $N$ is the number of molecules). It is experimentally accessible via incoherent inelastic neutron scattering experiments, ${ }^{24}$ and reflects the density of states (DOS) of a system without masking the various modes with their transition dipoles or transition polarizabilities, which typically vary for the different modes of a system.

Alternatively, one may write the linear response function as (see Appendix A 1):

$$
R_{1}(t)=-\frac{1}{m}\left\langle\frac{\partial q(t)}{\partial \dot{q}(0)}\right\rangle,
$$

which includes the stability matrix $\partial q(t) / \partial p(0)$. It can be calculated by numerical differentiation, i.e., by taking 
structural snapshots from an equilibrium MD simulation, perturbing the velocities of the individual degrees of freedom by plus/minus a small amount, and propagating the perturbed trajectories from there on along $t$. However, that procedure converges extremely slowly, hence Eq. 7 is not a very practical expression. The equivalence is nevertheless insightful, as the stability matrix is unavoidable for higher-order response functions, at least in principle (see Eq. 11 below).

\section{B. Nonlinear Response Functions}

Now turning to 2D-Raman (i.e., a Raman-RamanRaman (RRR) pulse sequence) ${ }^{16,22,25-41}$ and 2D-Raman$\mathrm{THz}$ hybrid spectroscopy with the Raman-THz-THz (RTT), THz-Raman-THz (TRT) ${ }^{4-7,42}$ and THz-THzRaman (TTR) ${ }^{43,44}$ pulse sequences, one needs to consider the following response functions: ${ }^{45}$

$$
\begin{aligned}
& R_{2}^{(\mathrm{RRR})}\left(t_{1}, t_{2}\right)=\left(\frac{i}{\hbar}\right)^{2} \operatorname{Tr}\left(\hat{\Pi}\left(t_{2}+t_{1}\right)\left[\hat{\Pi}\left(t_{1}\right),\left[\hat{\Pi}(0), \hat{\rho}_{\mathrm{eq}}\right]\right]\right) \\
& R_{2}^{(\mathrm{RTT})}\left(t_{1}, t_{2}\right)=\left(\frac{i}{\hbar}\right)^{2} \operatorname{Tr}\left(\hat{\mu}\left(t_{2}+t_{1}\right)\left[\hat{\mu}\left(t_{1}\right),\left[\hat{\Pi}(0), \hat{\rho}_{\mathrm{eq}}\right]\right]\right) \\
& R_{2}^{(\mathrm{TRT})}\left(t_{1}, t_{2}\right)=\left(\frac{i}{\hbar}\right)^{2} \operatorname{Tr}\left(\hat{\mu}\left(t_{2}+t_{1}\right)\left[\hat{\Pi}\left(t_{1}\right),\left[\hat{\mu}(0), \hat{\rho}_{\mathrm{eq}}\right]\right]\right) \\
& R_{2}^{(\mathrm{TTR})}\left(t_{1}, t_{2}\right)=\left(\frac{i}{\hbar}\right)^{2} \operatorname{Tr}\left(\hat{\Pi}\left(t_{2}+t_{1}\right)\left[\hat{\mu}\left(t_{1}\right),\left[\hat{\mu}(0), \hat{\rho}_{\mathrm{eq}}\right]\right]\right) .
\end{aligned}
$$

When recasting those in a DOS picture, in analogy to the linear response Eq. 3, three possibilities exist (the expression with $\hat{q}$ entering linear at all three points in time vanishes for isotropic systems): ${ }^{13,32}$

$$
\begin{aligned}
& R_{2}^{(1)}\left(t_{1}, t_{2}\right)=\left(\frac{i}{\hbar}\right)^{2} \operatorname{Tr}\left(\hat{q}\left(t_{2}+t_{1}\right)\left[\hat{q}\left(t_{1}\right),\left[\hat{q}^{2}(0), \hat{\rho}_{\mathrm{eq}}\right]\right]\right) \\
& R_{2}^{(2)}\left(t_{1}, t_{2}\right)=\left(\frac{i}{\hbar}\right)^{2} \operatorname{Tr}\left(\hat{q}\left(t_{2}+t_{1}\right)\left[\hat{q}^{2}\left(t_{1}\right),\left[\hat{q}(0), \hat{\rho}_{\mathrm{eq}}\right]\right]\right) \\
& R_{2}^{(3)}\left(t_{1}, t_{2}\right)=\left(\frac{i}{\hbar}\right)^{2} \operatorname{Tr}\left(\hat{q}^{2}\left(t_{2}+t_{1}\right)\left[\hat{q}\left(t_{1}\right),\left[\hat{q}(0), \hat{\rho}_{\mathrm{eq}}\right]\right]\right)
\end{aligned}
$$

where the source of the quadratic term $\hat{q}^{2}(t)$ can be either electrical or mechanical anharmonicity. ${ }^{13}$ Only $R_{2}^{(2)}\left(t_{1}, t_{2}\right)$ gives rise to an echo, since a quadratic term is needed in the middle of the pulse sequence for an "inversion of coherence" to occur. ${ }^{46}$ This can be seen from the corresponding coherence pathway:

$$
|0\rangle\langle 0|\stackrel{\hat{q}(0)}{\longrightarrow}| 0\rangle\left\langle 1\left|\stackrel{\hat{q}^{2}\left(t_{1}\right)}{\longrightarrow}\right| 2\right\rangle\left\langle 1\left|\stackrel{\hat{q}\left(t_{1}+t_{2}\right)}{\longrightarrow}\right| 1\right\rangle\langle 1|,
$$

with a $|0\rangle\langle 1|$-coherence during period $t_{1}$ and a $|2\rangle\langle 1|$ coherence during period $t_{2}$. For a close-to-harmonic oscillator, one has $\omega_{21} \approx-\omega_{01}$, hence fluctuations of the two frequencies will be correlated, but the sign switches. These two properties gives rise to rephasing in the presence of inhomogeneous broadening.

In the classical limit, this response function becomes (see Appendix A 2):

$$
R_{2}^{(2)}\left(t_{1}, t_{2}\right)=\frac{2}{k_{B} T}\left\langle\frac{\partial q\left(t_{1}+t_{2}\right)}{\partial p\left(t_{1}\right)} q\left(t_{1}\right) \dot{q}(0)\right\rangle,
$$

which again includes the stability matrix $\partial q\left(t_{1}+\right.$ $\left.t_{2}\right) / \partial p\left(t_{1}\right)$. Motivated by the equivalence of Eq. 4 and Eq. 7, the following correlation function is introduced:

$$
\left\langle q\left(t_{2}+t_{1}\right) \dot{q}\left(t_{1}\right) q\left(t_{1}\right) \dot{q}(0)\right\rangle
$$

in order to avoid the high numerical costs of calculating the stability matrix. The terms $q\left(t_{2}+t_{1}\right)$ and $q\left(t_{1}\right)$ are replaced by their time-derivatives to obtain the threepoint velocity autocorrelation function:

$$
c_{2}^{(2)}\left(t_{1}, t_{2}\right)=\left\langle v\left(t_{1}+t_{2}\right) v^{2}\left(t_{1}\right) v(0)\right\rangle .
$$

This is done in analogy to the velocity autocorrelation function Eq. 6; in either case, this modification suppresses the contribution from low frequency degrees of freedoms, in particular the diffusive motion in liquid water.

It is important to note that the step from Eq. 11 to Eq. 12 is not (!) rigorous (for a discussion of the differences and equivalences of Eq. 11 vs Eq. 12, see Appendix A 3). Nonetheless, Eq. 12 reveals an useful and at the same time very easy to calculate three-point correlation function. In particular, it contains the desired rephasing contribution, which can be seen when considering a single harmonic mode without dephasing, $v(t)=v_{0} \sin (\omega t)$. In this case, the corresponding correlation function is obtained by averaging over all phases $\phi$ :

$$
\begin{aligned}
c_{2}^{(2)}\left(t_{1}, t_{2}\right) & \propto \int_{0}^{2 \pi} \sin \phi \sin ^{2}\left(\omega t_{1}+\phi\right) \sin \left(\omega\left(t_{1}+t_{2}\right)+\phi\right) d \phi \\
& \propto \cos \left(\omega\left(t_{1}-t_{2}\right)\right)+2 \cos \left(\omega\left(t_{1}+t_{2}\right)\right),
\end{aligned}
$$

where the first term with opposite signs of frequency $\omega$ during period $t_{1}$ and $t_{2}$ will rephase and cause an "echo" at time $t_{1}=t_{2}$, i.e., that term will not cancel at time $t_{1}=$ $t_{2}$ when averaging over an ensemble of modes with an inhomogeneous distribution of frequencies $\omega$. In contrast, the second term will then decay very quickly as a function of time $t_{1}+t_{2}$.

As anticipated from Eq. 10, the other two possibilities:

$$
\begin{aligned}
c_{2}^{(1)}\left(t_{1}, t_{2}\right) & \propto \int_{0}^{2 \pi} \sin ^{2} \phi \sin \left(\omega t_{1}+\phi\right) \sin \left(\omega\left(t_{1}+t_{2}\right)+\phi\right) d \phi \\
& \propto \cos \left(\omega\left(2 t_{1}+t_{2}\right)\right)+2 \cos \left(\omega t_{2}\right) \\
c_{2}^{(3)}\left(t_{1}, t_{2}\right) & \propto \int_{0}^{2 \pi} \sin \phi \sin \left(\omega t_{1}+\phi\right) \sin ^{2}\left(\omega\left(t_{1}+t_{2}\right)+\phi\right) d \phi \\
& \propto \cos \left(\omega\left(t_{1}+2 t_{2}\right)\right)+2 \cos \left(\omega t_{1}\right)
\end{aligned}
$$

don't contain any rephasing term, hence only $c_{2}\left(t_{1}, t_{2}\right) \equiv$ $c_{2}^{(2)}\left(t_{1}, t_{2}\right)$ will be considered for the remainder of the paper.

\section{Model Based on Langevin Dynamics}

To demonstrate the information content of the twopoint and three-point velocity correlation functions, a 

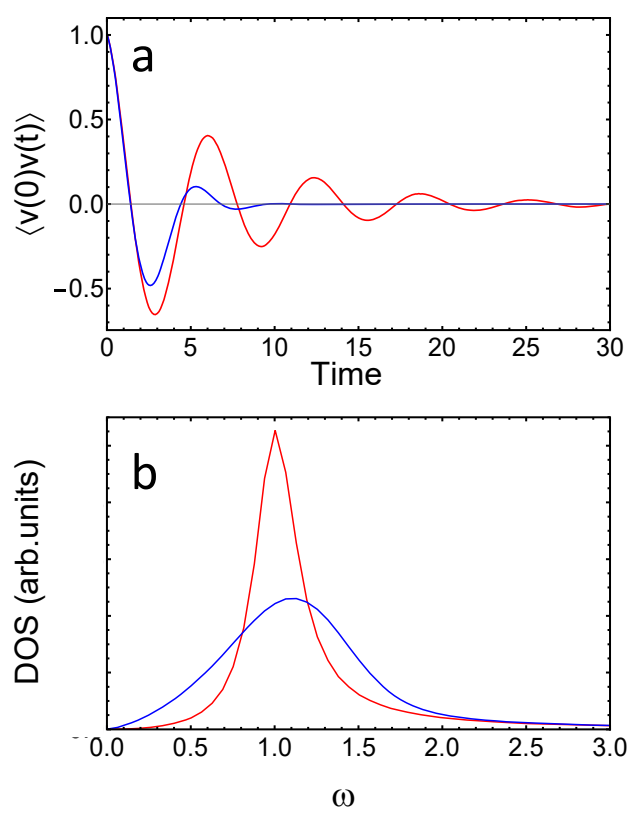

FIG. 1. (a) Velocity autocorrelation function of the Langevin model with a single oscillator with frequency $\omega=1$ (red), and an inhomogeneous ensemble of oscillators with Gaussian distributed force constants with $\delta k=0.7$ (blue). (b) The same after Fourier transformation (Eq. 5).

simple model is considered, i.e., a harmonic oscillator with Langevin dynamics. To that end,

$$
m \ddot{q}(t)=-k q(t)-m \gamma \dot{q}(t)+\delta F(t)
$$

is time-propagated, where $\delta F(t)$ is a fluctuating Gaussian distributed and $\delta$-correlated force, whose standard deviation is determined according to the fluctuationdissipation theorem:

$$
\delta F=\sqrt{2 m \gamma k_{B} T} .
$$

Unit-less constants with $k_{B} T=1$ and $m=1$ are used. The friction coefficient $\gamma$ determines the homogenous dephasing rate; $\gamma=0.3$ was used in the concrete example. The red plots in Fig. 1 show the velocity autocorrelation function in (a) the time and (b) the frequency domain for a single oscillator with force constant $k=1$ (revealing frequency $\omega=1$ ). The blue plots show the results when averaging over an inhomogeneous ensemble of such oscillators with Gaussian distributed force constants $k$ with standard deviation 0.7 (negative $k$ 's are discarded). As expected, the velocity autocorrelation function decays faster in the second case due to the additional inhomogenous dephasing. However, without assuming a model, it would not be possible to disentangle homogeneous from inhomogeneous dephasing based on these two-point correlation functions. ${ }^{47}$

With that, we turn to the three-point correlation function $c_{2}\left(t_{1}, t_{2}\right)$, showing its 2D Fourier transformation in Fig. 2. In the homogeneous case (Fig. 2a), two peaks can be identified, corresponding to the two terms of Eq. 14 .
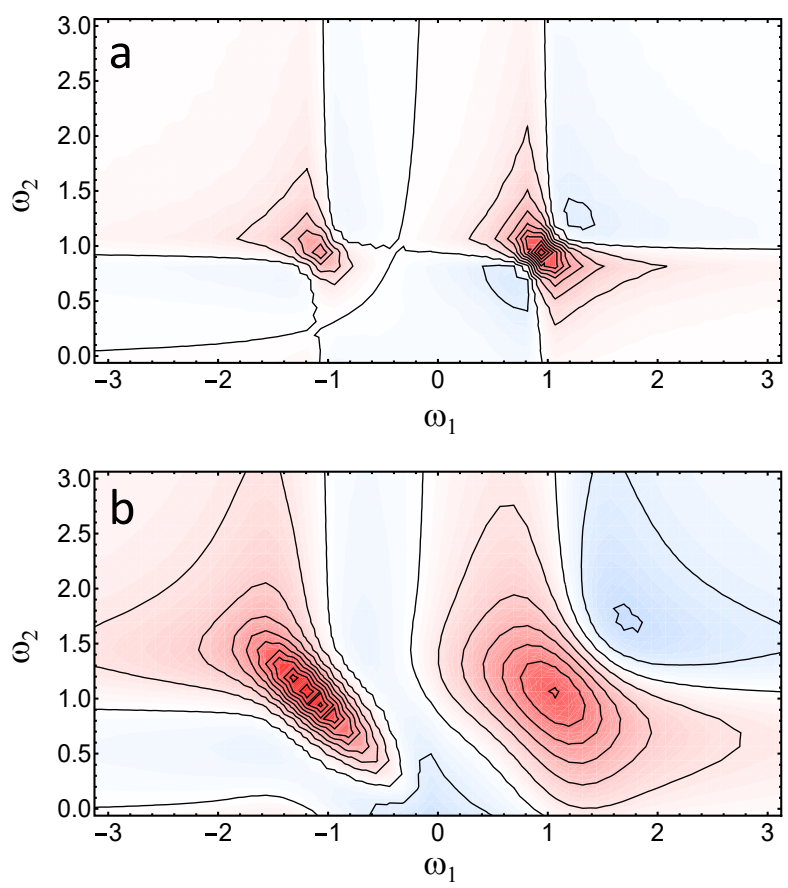

FIG. 2. 2D Fourier transformation (real part) of the threepoint correlation function $c_{2}\left(t_{1}, t_{2}\right)$ of (a) a homogeneous ensemble and (b) an inhomogeneous ensemble of oscillators (same parameters as in Fig. 1). Red and blue colors depict positive and negative signs of the response function.

The left quadrant is the rephasing quadrant with negative frequency $\omega_{1}$ and positive frequency $\omega_{2}$, see first term in Eq. 14. The peak in that quadrant is weaker by a factor 2 . Fig. $2 \mathrm{~b}$ shows the same for the inhomogeneous ensemble, in which case the rephasing peak is now the stronger one and becomes very elongated along the diagonal. As is well established in 2D spectroscopy, ${ }^{48}$ that elongation is the hallmark of inhomogenous broadening. It is the frequency-domain equivalent of an echo. However, as a caveat it important to note that also in the homogeneous case, the peak in the rephasing quadrant is slightly elongated along the diagonal (Fig. 2a). This is a consequence from the fact that these $2 \mathrm{D}$ peaks are "phase-twisted", and not "purely absorptive", as is common now in 2D spectroscopy. ${ }^{49}$

In equilibrium, the velocity $v(t)$ is Gaussian distributed, $\rho(v) \propto \exp \left(-m v^{2} / 2 k_{B} T\right)$. The width of that Gaussian distribution does not dependent of the potential energy function, hence it is Gaussian distributed in the homogeneous as well as in the inhomogeneous case. However, the fact that $v(t)$ is Gaussian distributed is only a necessary, and not a sufficient conditions for $v(t)$ being a "Gaussian process" ${ }^{50}$ A stochastic process is called Gaussian if $\left(v\left(t_{1}\right), v\left(t_{2}\right), \ldots, v\left(t_{n}\right)\right)$ is a multivariate Gaussian random variable for any finite set of times $\left(t_{1}, t_{2}, . . t_{n}\right) .{ }^{51}$ If $v(t)$ were a Gaussian process, the three-point correlation function $c_{2}\left(t_{1}, t_{2}\right)=$ $\left\langle v\left(t_{1}+t_{2}\right) v^{2}\left(t_{1}\right) v(0)\right\rangle$ would separate into two-point cor- 

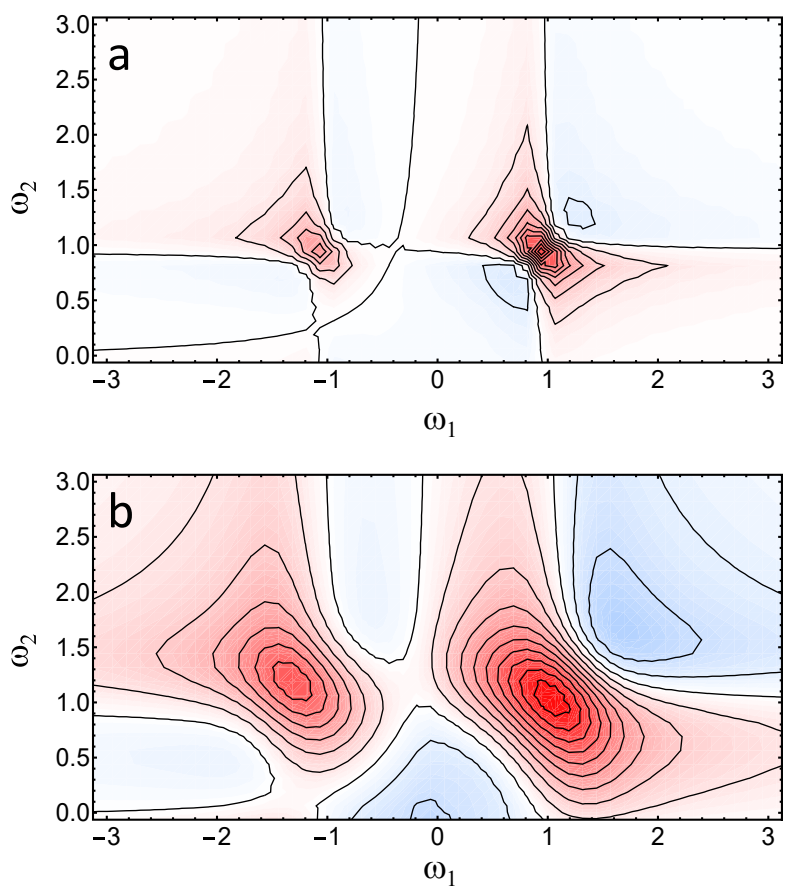

FIG. 3. The same as Fig. 2, but plotting $c_{2}^{\prime}\left(t_{1}, t_{2}\right)$ instead, which would be the same as $c_{2}\left(t_{1}, t_{2}\right)$ if $v(t)$ were a Gaussian stochastic process. Panel (a) is for a homogeneous ensemble, panel (b) an inhomogeneous ensemble of oscillators, and red and blue colors depict positive and negative signs of the response function.

relation functions, since higher than second-order cumulants vanish in this case: ${ }^{51}$

$$
c_{2}^{\prime}\left(t_{1}, t_{2}\right) \equiv 2\left\langle v\left(t_{2}\right) v(0)\right\rangle\left\langle v\left(t_{1}\right) v(0)\right\rangle+\left\langle v^{2}\right\rangle\left\langle v\left(t_{1}+t_{2}\right) v(0)\right\rangle
$$

and there would be no additional information in the $2 \mathrm{D}$ spectrum $c_{2}^{\prime}\left(\omega_{1}, \omega_{2}\right)$ that is not already contained in the 1D spectrum $c_{1}(\omega)$. It is illustrative to compare $c_{2}\left(\omega_{1}, \omega_{2}\right)$ and $c_{2}^{\prime}\left(\omega_{1}, \omega_{2}\right)$ for the two cases, see Fig. 3. The 2D spectra are indistinguishable in the homogeneous case, reflecting the the Gaussian statistics of the Langevin equation itself. Both do however differ in the inhomogenous case, in which case the antidiagonal of the rephasing peak of $c_{2}^{\prime}\left(\omega_{1}, \omega_{2}\right)$ is wider than that of $c_{2}\left(\omega_{1}, \omega_{2}\right)$, hence $c_{2}^{\prime}\left(\omega_{1}, \omega_{2}\right)$ looks like that of a homogenous ensemble with a faster decay time. The comparison of $c_{2}^{\prime}\left(\omega_{1}, \omega_{2}\right)$ and $c_{2}\left(\omega_{1}, \omega_{2}\right)$ can therefore serve as a measure of the amount of inhomogeneous contribution in a velocity $2 \mathrm{D}$ spectrum.

\section{METHODS: MD SIMULATIONS}

Molecular dynamics (MD) simulations were performed in Gromacs, ${ }^{52}$ using the TIP4P/2005 water model with periodic boundary conditions. ${ }^{53}$ If not mentioned otherwise, a large simulation box with 5000 water molecules was used to minimize limited size effects. Time steps were $2 \mathrm{fs}$, the Lennard Jones interactions were switched to zero form $0.9 \mathrm{~nm}$ to $1.0 \mathrm{~nm}$ and the long range electrostatic forces approximated by the Particle-Mesh-Ewald approximation. ${ }^{54}$ For a comparison, a box with only 100 water molecules was also considered, in which case the Lennard Jones interactions were switched to zero form $0.61 \mathrm{~nm}$ to $0.71 \mathrm{~nm}$. To determine the box-size, the simulation box has been pre-equilibrated for $10 \mathrm{~ns}$ in the $N p T$ ensemble at 1 bar and $290 \mathrm{~K}$, using velocity-rescaling ${ }^{55}$ and the Berendsen barostat. ${ }^{56}$ For the subsequent equilibration in the $N V T$ ensemble (2 ns), the averaged box size from the previous $N p T$ simulation was used. Production runs were performed in the NVT ensemble for $10 \mathrm{~ns}$ with saving time 4 fs.

For the simulations of supercooled water and amorphous ice, snapshot structures were sampled every 10 ps from an equilibrium trajectory of neat water at $290 \mathrm{~K}$, which were then annealed by linearly ramping down the temperature in the $N p T$ ensemble to the desired temperature with a cooling rate of $1 \mathrm{~K} / \mathrm{ps}$. At each temperature, 100 such boxes were generated, that were then simulated for 100 ps in the $N V T$ ensemble for the production runs (i.e., total simulation time again $10 \mathrm{~ns}$ ).

As in the experimental work of aqueous salt solutions, ${ }^{6}$ $2 \mathrm{M}$ of $\mathrm{CsCl}, \mathrm{NaCl}, \mathrm{SrCl}_{2}$ or $\mathrm{MgCl}_{2}$ (i.e., 182 cations and the corresponding number of $\mathrm{Cl}^{-}$ions) was added to the water box by randomly replacing water molecules. The OPLS $^{57}$ parameters were used for the charges and the Lennard Jones interactions of the ions, as implemented in Gromacs ${ }^{52}$ (see Table I). The subsequent equilibration was performed in the same way as described above for neat water.

\section{RESULTS AND DISCUSSION}

\section{A. Neat Water}

Fig. 4 shows the oxygen velocity two-point correlation function of neat water at $290 \mathrm{~K}$, revealing in essence two peaks, one around $50 \mathrm{~cm}^{-1}$, which is typically assigned to a hydrogen-bond bend vibration, and the other around $250 \mathrm{~cm}^{-1}$, the hydrogen-bond stretch vibration. It is worth noting that TIP4P/2005, despite the fact that it completely fails to reproduce the second band in the $\mathrm{THz}$ spectrum of water, of course contains that degree

TABLE I. Ion parameters used in the simulations, taken from the OPLS ${ }^{57}$ implementation of Gromacs. ${ }^{52}$

\begin{tabular}{l|lll} 
& $q(e)$ & $\sigma(\AA)$ & $\epsilon(\mathrm{kJ} / \mathrm{mol})$ \\
\hline $\mathrm{Cl}^{-}$ & -1 & 4.41724 & 0.49283 \\
$\mathrm{Cs}^{+}$ & +1 & 6.71600 & 0.00034 \\
$\mathrm{Na}^{+}$ & +1 & 3.33045 & 0.01160 \\
$\mathrm{Mg}^{+}$ & +2 & 1.64447 & 3.66118 \\
$\mathrm{Sr}^{+}$ & +2 & 3.10269 & 0.49466
\end{tabular}



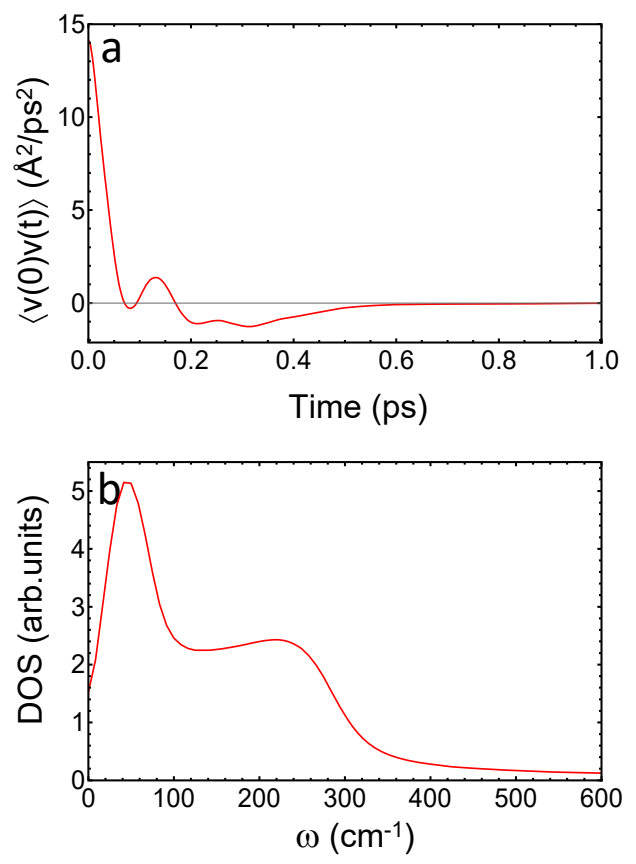

FIG. 4. Oxygen velocity autocorrelation function of neat water at $290 \mathrm{~K}$ in (a) the time and (b) the frequency domain.
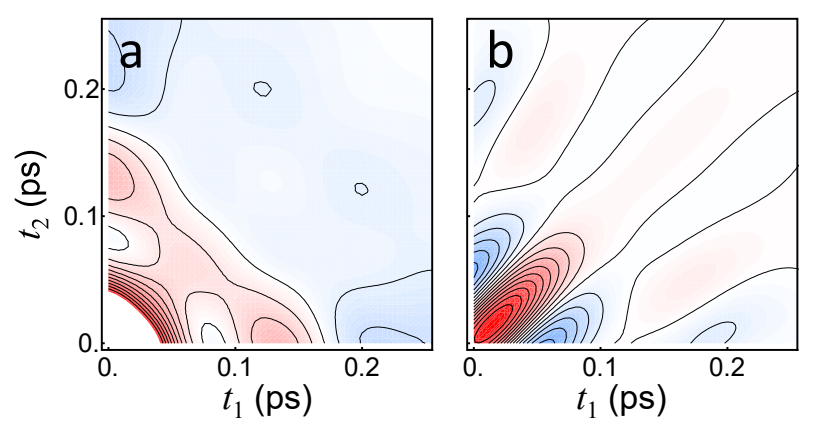

FIG. 5. (a) Three-point correlation function $c_{2}\left(t_{1}, t_{2}\right)$ of neat water at $290 \mathrm{~K}$ and (b) its isolated rephasing contribution (see text for details). Red and blue colors depict positive and negative signs of the response function.

of freedom (yet, it overestimates a bit its frequency). TIP4P/2005 water doesn't give that mode any transition dipole, since it consists of fixed point charges and hence the vibration of two water molecules against each other does not change their dipoles. It has been shown that the intensity of that band is related to inter-molecular charge transfer upon hydrogen bonding. ${ }^{58-60}$

On the other hand, the third prominent mode in the $\mathrm{THz}$ spectrum of water, the librational mode around $600 \mathrm{~cm}^{-1}$, is essentially missing in the oxygen velocity autocorrelation function. It is missing since the rotation of a water molecule around its center-of-mass barely affects the position of the oxygen (the band is present in the hydrogen velocity autocorrelation function, ${ }^{24}$ which is however not considered here).

The corresponding three-point correlation function
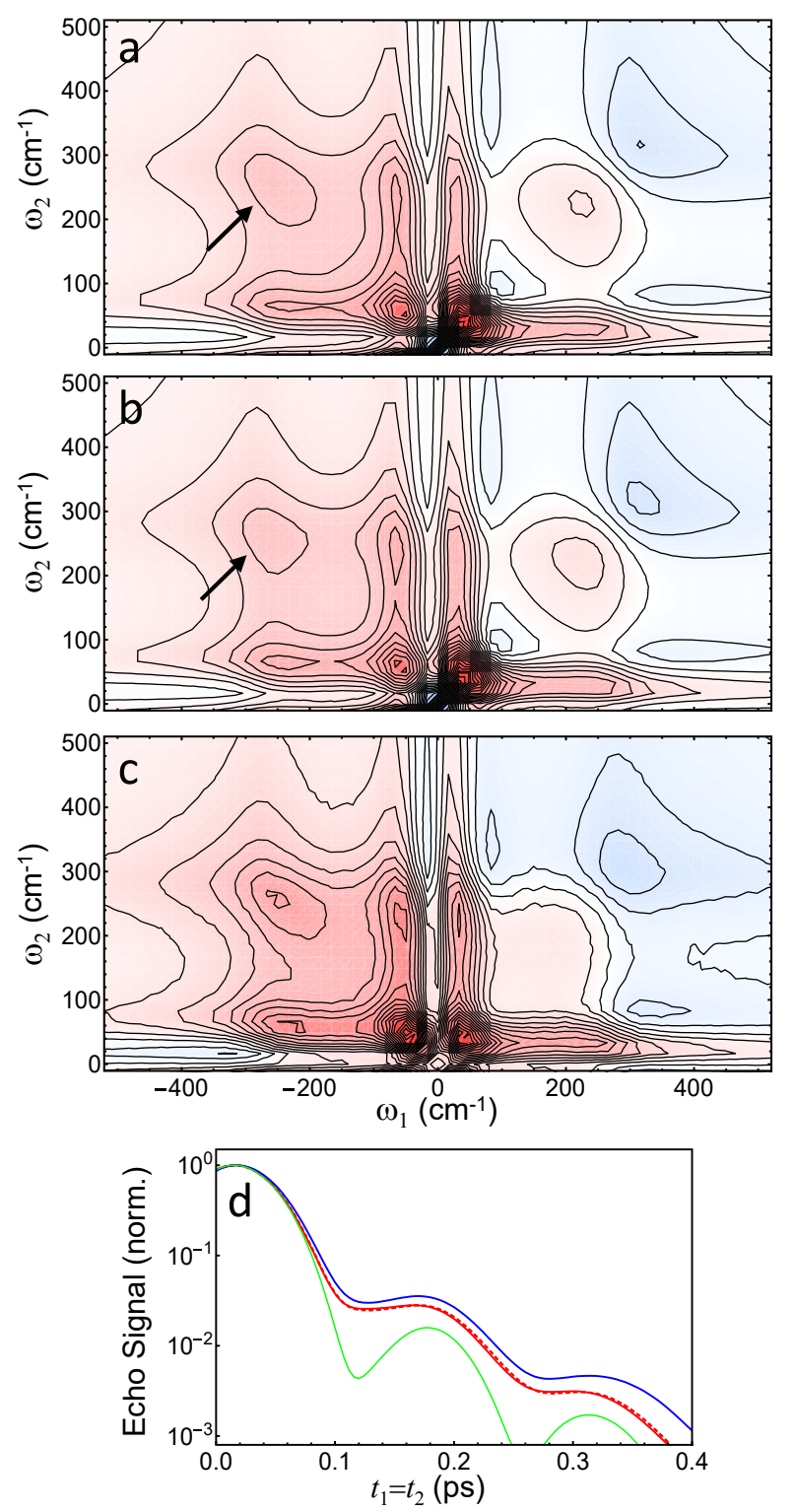

FIG. 6. (a) Fourier transformation of $c_{2}\left(t_{1}, t_{2}\right)$, (b) of $c_{2}^{\prime}\left(t_{1}, t_{2}\right)$, and (c) of Eq. 11, all for neat water at $290 \mathrm{~K}$. Red and blue colors depict positive and negative signs of the response function. The arrows in panels (a, b) mark the rephasing peak of the hydrogen-bond stretch vibration. (d) Echo signal taken as $t_{1}=t_{2}$-cut from the isolated rephasing contribution: (red, solid line) for $c_{2}\left(t_{1}, t_{2}\right)$ and a simulation box with 5000 water molecules, (red, dashed) for 100 water molecules, green for $c_{2}^{\prime}\left(t_{1}, t_{2}\right)$ and blue for Eq. 11 .

$c_{2}\left(t_{1}, t_{2}\right)$ is shown in Fig. 5a together with its Fourier transformation $c_{2}\left(\omega_{1}, \omega_{2}\right)$ in Fig. 6a. The discussion focuses on the (left) rephasing quadrant of the 2D spectrum (Fig. 6a), where two diagonal peaks can be identified that correspond to the two peaks in the $1 \mathrm{D}$ spectrum (Fig. 4b). In addition, cross peaks show up, indicating the coupling between both degrees of freedom. In particular the peak related to the hydrogen-bond stretch 
vibration around $250 \mathrm{~cm}^{-1}$ is elongated along the diagonal. Comparison with Fig. 6b shows that this elongation is a bit more pronounced than for $c_{2}^{\prime}\left(\omega_{1}, \omega_{2}\right)$ (compare the two peaks marked by an arrow), indicating that the band is inhomogeneously broadened to a certain extent.

In order to quantify the echo lifetime, the rephasing signal has been isolated by masking the $2 \mathrm{D}$ signal with a filter function $\exp \left[-\left(\left(\omega_{1}+\omega_{0}\right)^{4}+\left(\omega_{2}-\omega_{0}\right)^{4}\right) / d \omega^{4}\right]$ with $\omega_{0}=280 \mathrm{~cm}^{-1}$ and $d \omega=215 \mathrm{~cm}^{-1}$, and Fouriertransforming the data back into the time domain (see Fig. 5b). Fig. 6d (red) shows the diagonal cut along $t_{1}=t_{2}$ of these data. An initial spike is observed that lasts for up to $100 \mathrm{fs}$, followed by a beating signal that is superimposed on an exponentially decaying wing. The frequency of the beating signal $\left(\approx 200 \mathrm{~cm}^{-1}\right)$ coincides with the splitting between the two bands in the linear spectrum, and thus is related to the coupling between them. In the case of $c_{2}^{\prime}\left(\omega_{1}, \omega_{2}\right)$ (Fig. 6d, green), the initial spike and the beating contribution are similar, but the exponential wing on which the latter is residing is significantly reduced.

Due to the complicated decay of the echo signal, no attempts have been made to fit it to a proper functional form. Instead, the "average echo decay time" has bee calculated, defined as:

$$
\langle\tau\rangle=\frac{\int_{0}^{\infty} \tau \cdot c_{2}(\tau, \tau) d \tau}{\int_{0}^{\infty} c_{2}(\tau, \tau) d \tau}
$$

revealing 34.4 fs for $c_{2}\left(t_{1}, t_{2}\right)$ (Fig. $6 \mathrm{~d}$, red, solid), as opposed to 27.5 fs for $c_{2}^{\prime}\left(t_{1}, t_{2}\right)$ (Fig. $6 \mathrm{~d}$, green). That small difference quantifies the amount of inhomogeneous broadening in liquid TIP4P / 2005 water at room temperature.

To test the effect of the empirical step from Eq. 11 to Eq. 12, the former has been calculated as well by numerical differentiation of the stability matrix (see Fig. 6c). In order to be able to compare it directly to $c_{2}\left(t_{1}, t_{2}\right)$, the derivative with respect to $t_{2}$ was taken and the middle term of Eq. 11, $q\left(t_{1}\right)$, was replaced by its time-derivative as well. This modification is equivalent to that from Eq. 12 to Eq. 13. Furthermore, to keep the numerical effort affordable, the size of the simulation box had to be reduced to 100 water molecules in this case. The total simulation time for Fig. 6c has been 20'000 times longer than that for Fig. 6a (albeit for a box that is 50 times smaller), yet the signal-to-noise ratio is much worse, illustrating the huge numerical advantage of Eq. 13. Independent of that, however, the two 2D spectra are very similar to each other, the major difference being the nonrephasing peak, that is smaller in Fig. 6c in a relative sense, which originates from the additional term Eq. A16. The echo decay is slowed down a bit in Eq. 11 (compare Fig. 6d, red vs green) with average decay times of 38.9 fs vs 34.4 fs. It has been verified that this difference does not originate from the different sizes of the simulation boxes by also calculating $c_{2}\left(t_{1}, t_{2}\right)$ for 100 water molecules, which introduces only minor changes (see Fig. 6d, red, solid vs dashed lines).
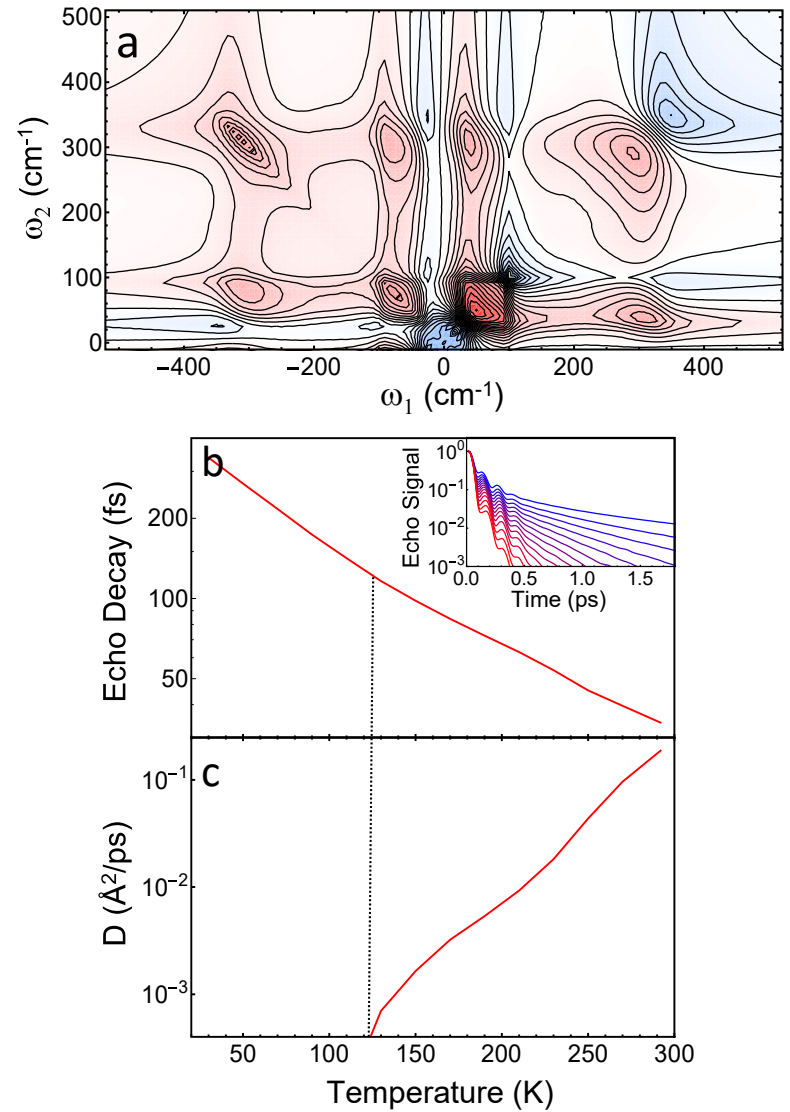

FIG. 7. (a) Fourier transformation of the three-point correlation function $c_{2}\left(t_{1}, t_{2}\right)$ of amorphous ice at $90 \mathrm{~K}$. Red and blue colors depict positive and negative signs of the response function. (b) Averaged echo decay time as a function of temperature; the corresponding echo signals are shown in the inset from $30 \mathrm{~K}$ (blue) to $290 \mathrm{~K}$ (red) in steps of $30 \mathrm{~K}$. (c) Selfdiffusion coefficient as a function of temperature. The vertical dotted line marks the glass transition, where self-diffusion essentially ceases.

\section{B. Temperature Dependence}

Starting with amorphous ice as a limiting case, Fig. 7a shows the Fourier transformation of the three-point correlation function $c_{2}\left(t_{1}, t_{2}\right)$ at $90 \mathrm{~K}$. In that case, the anti-diagonal peak of the hydrogen-bond stretch mode in the rephasing quadrant is significantly more narrow than in liquid water (Fig. 6a), despite the fact the diagonal widths are very much comparable. The average echo decay time, calculated in the same way as for liquid water, is $170 \mathrm{fs}$ at $90 \mathrm{~K}$.

Fig. 7b explores the echo decay in a wide temperature range from ambient conditions $(290 \mathrm{~K}$, same as in Fig. 6) through supercooled water into the regime of amorphous ice down to $30 \mathrm{~K}$. Above freezing point, which is the temperature range investigated experimentally, ${ }^{7}$ the echo-decay time increases with lowering the temperature, but the effect is quite weak with only about 
$-0.7 \% / \mathrm{K}$, even less than in experiment $(-1.7 \% / \mathrm{K}) .{ }^{7}$ As expected, the trend continues without change into the supercooled regime. The self-diffusion coefficient shown in Fig. $7 \mathrm{c}$, calculated as: ${ }^{61}$

$$
D=\int_{0}^{\infty}\langle v(t) v(0)\rangle d \tau
$$

suggests that the glass transition occurs at about $120 \mathrm{~K}$ for the TIP4P/2005 water model under the chosen cooling conditions (see Sec. II), which is when self-diffusion ceases. At this temperature, the averaged echo time exhibits a minor kink (marked by the vertical dashed line), and the slope becomes a bit steeper below the glass transition.

One could have expected that the echo lifetime diverges as one approaches the glass transition, since amorphous ice is structurally heterogeneous on essentially infinite timescales. In contrary to that expectation, the averaged echo lifetime increases in a continues fashion with a barely visible kink as one crosses the glass transition. One can conclude from these observations that the major effect that determines the echo lifetime is actually the anharmonicity of the intermolecular modes, and not the structural flexibility of the sample per se. That is, a set of harmonic modes would decouple completely in a normal mode representation, and Eq. 14 would apply strictly for each one of these normal modes. Integrating Eq. 14 over a distribution of normal modes would reveal an echo on infinite timescales. That is no longer true for anharmonic modes; in that case, there is no representation equivalent to a normal mode represntation, in which degrees of freedom decouple. Since there is still thermal noise at any finite temperature, thermal fluctuations of one mode will cause fluctuating frequency shifts, and hence dephasing, of other modes via anharmonic coupling. Hence, despite the fact that the set of modes is stationary in amorphous ice, the terms in Eg. 14 would have to be amended with a homogeneous dephasing term that renders the echo lifetime finite. However, as one cools down amorphous ice further below the glass transition, thermal fluctuations explore less of their amharmonic potential, hence the effect of anharmonic dies out and the echo lifetime increases further. In the limit $T \rightarrow 0$, but only in that limit, the echo lifetimes is expected to indeed diverge.

One question however remains: In the regime below the glass transition, one would expect that only anharmonicity determines the echo lifetime, while both anharmonicity as well as structural flexibility should contribute above. The latter changes the hydrogen bond networks, and hence the constitution of the intramolecular modes. Given that the lifetime of a particular hydrogen bond is in the order of $1 \mathrm{ps}$ at room temperature ${ }^{62-66}$ and that such hydrogen networks over which intramolecular modes are delocalized consist of more than just one hydrogen bonds, the lifetime of these modes can be quite short and is expected to contribute to the fast decay of the echo. However, in that scenario, one would expect the temperature dependence to be steeper above the glass transition,
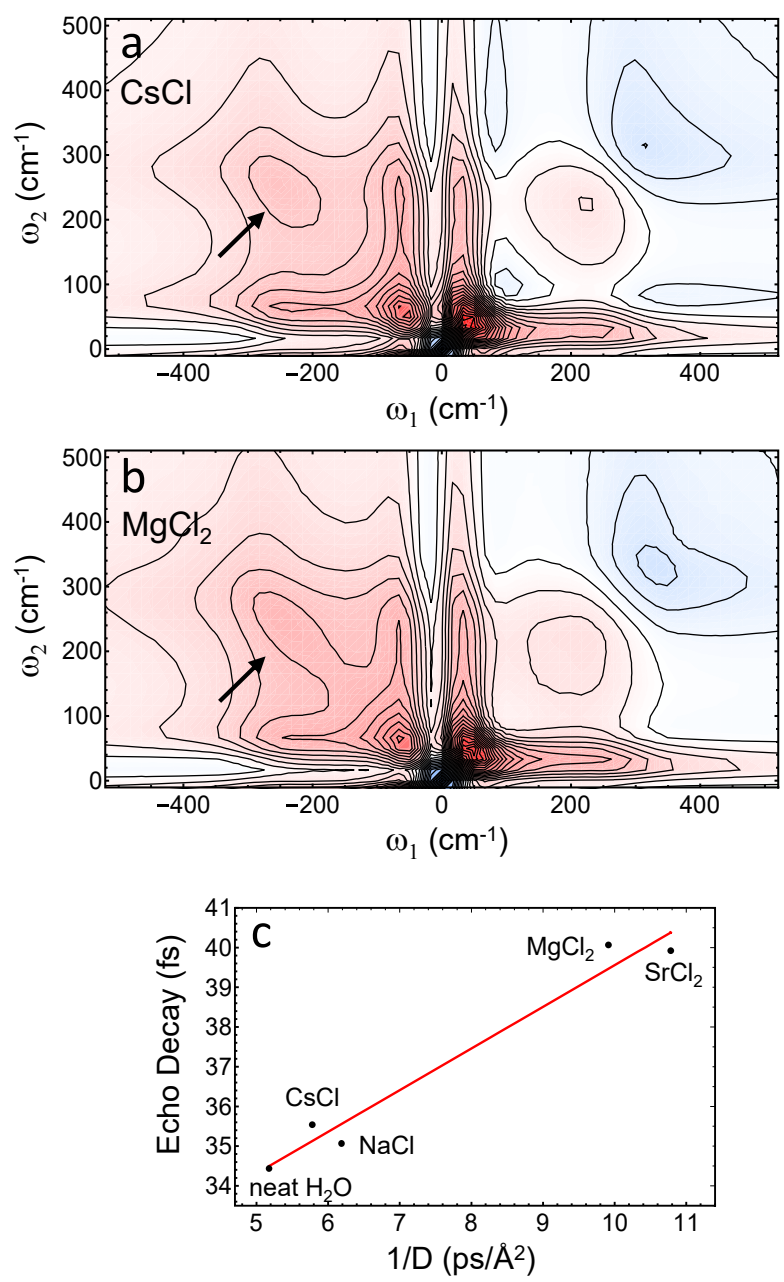

FIG. 8. Fourier transformation of the three-point correlation function $c_{2}\left(t_{1}, t_{2}\right)$ of (a) a $2 \mathrm{M} \mathrm{CsCl} \mathrm{solution} \mathrm{and} \mathrm{(b)} \mathrm{a} 2 \mathrm{M}$ $\mathrm{MgCl}_{2}$ solution at $290 \mathrm{~K}$. The arrows mark the rephasing peak of the hydrogen-bond stretch vibration. Red and blue colors depict positive and negative signs of the response function. (c) Echo decay time of neat water and various salt solutions plotted against the inverse of the self-diffusion constant, which is used here as a measure of viscosity.

in contrary to what is observed in Fig. 7b. The reason for that discrepancy is currently not clear and requires further investigation.

\section{Aqueous Salt Solutions}

Experimentally, the addition of certain salts has revealed a significantly extended echo as compared to neat water, and we had found a strong correlation between the echo lifetime and the extent to which a certain salt changes the viscosity of the solution. ${ }^{6}$ Fig. 8 explores whether these results are reproduced with the help of velocity echoes. The rephasing peak of the hydrogen-bond stretch vibration is significantly more extended along the 
diagonal for a $\mathrm{MgCl}_{2}$ solution than for a $\mathrm{CsCl}$ solution (compare Fig. 8a to Fig. 8b), and also in comparison to neat water (compare to Fig. 6). Fig. 8c plots the averaged echo decay time as a function of the inverse self-diffusion constant of water (calculated from Eq. 20), which is used here as measure of the viscosity of the solution. The same strong correlation is observed as in experiment, ${ }^{6}$ but the size of the effect is smaller, i.e. only a ca. $16 \%$ increase in echo decay time for a $2 \mathrm{M} \mathrm{SrCl}_{2}$ solution relative to neat water in the simulation vs $46 \%$ in experiment. On the other hand, the effect on the viscosity is overestimated with a ca. $100 \%$ increase in the simulation vs $50 \%$ in experiment, again for $2 \mathrm{M} \mathrm{SrCl}_{2}$. It needs be added that the quite simplistic point-charge model of the ions used here (Table I) cannot reproduce the structure breaking capability of $\mathrm{Cs}^{+}, 67$ that actually decreases the viscosity in experiment relative to neat water, and that the four cations considered here group essentially according to their charge (i.e., $\mathrm{Cs}^{+}$and $\mathrm{Na}^{+} \mathrm{vs}^{2+}$ and $\mathrm{Sr}^{2+}$ ).

\section{CONCLUSION}

The three-point correlation function $c_{2}\left(t_{1}, t_{2}\right)$ provides a very easy to calculate quantity that can measure the degree of inhomogeneous broadening in the density of states of a liquid by revealing echoes. The correlation function converges extremely quickly and allows one to observe echoes over 3 orders of magnitudes (Fig. 6d) with only $10 \mathrm{~ns}$ of simulation time of a box with 5000 water molecules; for a simple water model like TIP4P/2005 a matter of a few hours on a very modest computer. On the level of the contour plots of Fig. 6a, noise is basically not visible any more after 1 ns of simulation time. Such simulation times are feasible for essentially any model of water, including DFT water or ab initio derived water models. ${ }^{15}$ The three-point correlation function can readily be calculated from an equilibrium trajectory, which is the major reason for its fast convergence. That is seen from the comparison of Eq. 11 vs Eq. 12 (Fig. 6c). The former can be derived in a rigorous way from the corresponding quantum correlation function, however it converges significantly slower. The calculation of Eq. 11 requires effectively non-equilibrium calculations, which converge as slowly as the original correlation functions Eq. 8 needed for the description hybrid 2D-Raman- $\mathrm{THz}$ spectroscopy. ${ }^{14,16,17,42}$

Despite the fact that the three-point velocity correlation function cannot be compared one-to-one to an experimental $2 \mathrm{D}$ spectrum (either a 2D-Raman spectrum or a 2D-Raman-THz hybrid spectrum), since the information on transition dipoles and transition polarizabilities is not included, measured echo life times and their dependence on certain parameters can still be compared to a MD simulation. The experimentally measured temperature trend from room temperature down to the freezing point $^{7}$ and the correlation with viscosity upon addition of salts ${ }^{6}$ could be reproduced, see Figs. $7 \mathrm{~b}$ and $8 \mathrm{c}$, respec- tively. However, the numbers don't agree quantitatively; that is, the echo decay times are faster by a factor $2-3$ as compared to experiment, and also the trends, in a relative sense, are smaller by about the same factor. The non-rigorous step from Eq. 11 to Eq. 12 prolongs the lifetime a little bit, but not to the extent that it would fix the problem. Other possible explanations for this deficiency include the force field, which is something that can be tested, as the simulation time needed to converge the three-point velocity correlation function is very modest. Finally, it could be an artifact of classical mechanics. The frequency of the hydrogen bond stretch vibration (around $250 \mathrm{~cm}^{-1}$ ) is the same range as $k_{B} T$, hence it is not strictly a classical mode, and that is in particular true considering that the echo sequence includes a 2-quantum transition (Eq. 10). One might assume that the discreteness of quantum states reduces the contribution of anharmonic couplings to dephasing. Adding quantum effects to a water simulation by ring-polymer molecular dynamics (RPMD) ${ }^{68}$ might address that issue, however, the correct way to calculate higher-order correlation functions from RPMD is still an open issues. To test the dependence of the echo decay time on the isotope constitution of water, as explored experimentally as well, ${ }^{7}$ will also require the addition of quantum effects.

In conclusion, this work is a significant step towards a better understanding of recent 2D-Raman- $\mathrm{THz}$ experiments, ${ }^{5-7}$ which as of now provide the most detailed spectroscopic view into the intermolecular degrees of freedom of water.

\section{ACKNOWLEDGEMENT}

The idea for this work was born based on a spot-on question of Jeremy Richardson on our experimental results, and he also provided the derivation of Eqs. A6, A12 and A14-A17. Valuable discussions with Gerhard Stock and Yoshitaka Tanimura are acknowledged as well. The work has been supported by the Swiss National Science Foundation (SNF) through the National Center of Competence and Research (NCCR) MUST as well by the MaxWater network.

\section{Appendix A: Derivation of Response Functions in the Classical Limit}

\section{Linear Response}

In the classical limit, the commutator in Eq. 3 is replaced by a Poisson bracket, $1 / i \hbar[. ., ..] \rightarrow\{. . . .\}:.^{23}$

$$
R_{1}(t)=\left\langle q(t)\left\{q(0), \rho_{\mathrm{eq}}\right\}\right\rangle
$$


To derive Eq. 4 from that, the classical counterpart of the equilibrium density matrix is first specified:

$$
\rho_{e q}(\boldsymbol{p}, \boldsymbol{q})=\frac{1}{Z} e^{-\frac{p^{2}}{2 m k_{B} T}} e^{-\frac{V(\boldsymbol{q})}{k_{B} T}}
$$

where $V(\boldsymbol{q})$ is the potential energy that is a function of positions only, and $Z$ is a normalization factor (i.e., the partition function). With that, the Poisson bracket in Eq. A1 for one particular degree of freedom $i$ is (eventually, the average over $i$ will be taken):

$$
\begin{aligned}
& \left\{q_{i}(0), \rho_{\mathrm{eq}}(\boldsymbol{p}, \boldsymbol{q})\right\} \\
& \quad=\sum_{j} \frac{\partial q_{i}(0)}{\partial q_{j}(0)} \frac{\partial \rho_{\mathrm{eq}}(\boldsymbol{p}, \boldsymbol{q})}{\partial p_{j}(0)}-\frac{\partial q_{i}(0)}{\partial p_{j}(0)} \frac{\partial \rho_{\mathrm{eq}}(\boldsymbol{p}, \boldsymbol{q})}{\partial q_{j}(0)} \\
& \quad=-\frac{p_{i}(0)}{m} \frac{1}{k_{B} T} \rho_{\mathrm{eq}}(\boldsymbol{p}, \boldsymbol{q})=-\frac{1}{k_{B} T} \dot{q}_{i}(0) \rho_{\mathrm{eq}}(\boldsymbol{p}, \boldsymbol{q}) .
\end{aligned}
$$

The second term of the Poisson bracket vanishes, since $\partial q_{i}(0) / \partial p_{j}(0)=0$. Putting everything together, Eq. 4 is obtained.

Eq. 7 is obtained by first reshuffling the commutator:

$$
\begin{aligned}
R_{1}(t) & =-\frac{i}{\hbar} \operatorname{Tr}\left(q(t)\left[q(0), \rho_{\mathrm{eq}}\right]\right) \\
& =-\frac{i}{\hbar} \operatorname{Tr}\left([q(t), q(0)] \rho_{\mathrm{eq}}\right),
\end{aligned}
$$

which can be seen when evaluating the commutators and making use of the invariance of the trace under cyclic permutation. In the classical limit, the corresponding Poisson bracket is:

$$
\left\{q_{i}(t), q_{i}(0)\right\}=-\sum_{j} \frac{\partial q_{i}(t)}{\partial p_{j}(0)} \frac{\partial q_{i}(0)}{\partial q_{j}(0)}=-\frac{\partial q_{i}(t)}{\partial p_{i}(0)}
$$

The other term of the Poisson bracket vanishes since $\partial q_{i}(0) / \partial p_{j}(0)=0$.

Alternatively, on may start from Eq. 7 and integrate in parts:

$$
\begin{aligned}
\left\langle\frac{\partial q(t)}{\partial p(0)}\right\rangle & =\int \frac{\partial q(t)}{\partial p(0)} e^{-\frac{p(0)^{2}}{2 m k^{T}}-\frac{V(q(0))}{k_{B} T}} d p(0) d q(0) \\
& =-\int q(t) \frac{\partial}{\partial p(0)} e^{-\frac{p(0)^{2}}{2 m k_{B} T}-\frac{V(q(0)}{k_{B} T}} d p(0) d q(0) \\
& =\frac{1}{m k_{B} T} \int q(t) p(0) e^{-\frac{p(0)^{2}}{2 m k_{B} T}-\frac{V(q(0))}{k_{B} T}} d p(0) d q(0) \\
& =\frac{1}{m k_{B} T}\langle q(t) p(0)\rangle
\end{aligned}
$$

Classical approximations taken on equivalent quantummechanical expressions (i.e., Eqs. 3 and A4) may sometimes give different results, but not in this case.

\section{Nonlinear Response}

For the nonlinear response function, $R_{2}^{(2)}\left(t_{1}, t_{2}\right)$ from Eq. 9 is first rewritten in the following form:

$$
R_{2}^{(2)}\left(t_{1}, t_{2}\right)=\left(\frac{i}{\hbar}\right)^{2} \operatorname{Tr}\left(\left[\hat{q}\left(t_{2}+t_{1}\right), \hat{q}^{2}\left(t_{1}\right)\right]\left[\hat{q}(0), \hat{\rho}_{\mathrm{eq}}\right]\right)
$$

using the same trick as in Eq. A4. In the classical limit, this translates into: ${ }^{22}$

$$
R_{2}^{(2)}\left(t_{1}, t_{2}\right)=-\frac{1}{k_{B} T}\left\langle\left\{q\left(t_{2}+t_{1}\right), q^{2}\left(t_{1}\right)\right\} \dot{q}(0)\right\rangle
$$

where Eq. A3 has been used for the second Poisson bracket. For one particular degree of freedom $i$, one gets for the remaining Poisson bracket:

$$
\begin{aligned}
& \left\{q_{i}\left(t_{2}+t_{1}\right), q_{i}^{2}\left(t_{1}\right)\right\} \\
& =\sum_{j} \frac{\partial q_{i}\left(t_{1}+t_{2}\right)}{\partial q_{j}\left(t_{1}\right)} \frac{\partial q_{i}^{2}\left(t_{1}\right)}{\partial p_{j}\left(t_{1}\right)}-\frac{\partial q_{i}\left(t_{1}+t_{2}\right)}{\partial p_{j}\left(t_{1}\right)} \frac{\partial q_{i}^{2}\left(t_{1}\right)}{\partial q_{j}\left(t_{1}\right)} \\
& =-2 \frac{\partial q_{i}\left(t_{1}+t_{2}\right)}{\partial p_{i}\left(t_{1}\right)} q_{i}\left(t_{1}\right),
\end{aligned}
$$

which is since $\partial q_{i}^{2}\left(t_{1}\right) / \partial p_{j}\left(t_{1}\right)=0$ and $\partial q_{i}^{2}\left(t_{1}\right) / \partial q_{j}\left(t_{1}\right)=$ $2 q_{i} \delta_{i j}$. Putting everything together, Eq. 11 is obtained.

\section{Comparison of Eq. 11 vs Eq. 12}

There are various limiting cases, in which the differences and equivalences of Eq. 11 vs Eq. 12 can be expressed analytically. For example, one can separate the three-point correlation function Eq. 11 in the following way: ${ }^{51}$

$$
\begin{aligned}
\left\langle\frac{\partial q\left(t_{1}+t_{2}\right)}{\partial p\left(t_{1}\right)} q\left(t_{1}\right) \dot{q}(0)\right\rangle= & \left\langle\frac{\partial q\left(t_{1}+t_{2}\right)}{\partial p\left(t_{1}\right)} q\left(t_{1}\right) \dot{q}(0)\right\rangle_{C} \quad(\mathrm{~A} 10) \\
& +\left\langle\frac{\partial q\left(t_{1}+t_{2}\right)}{\partial p\left(t_{1}\right)}\right\rangle\left\langle q\left(t_{1}\right) \dot{q}(0)\right\rangle \\
& +\left\langle\frac{\partial q\left(t_{1}+t_{2}\right)}{\partial p\left(t_{1}\right)} q\left(t_{1}\right)\right\rangle\langle\dot{q}(0)\rangle \\
& +\left\langle\frac{\partial q\left(t_{1}+t_{2}\right)}{\partial p\left(t_{1}\right)} \dot{q}(0)\right\rangle\left\langle q\left(t_{1}\right)\right\rangle \\
& -2\left\langle\frac{\partial q\left(t_{1}+t_{2}\right)}{\partial p\left(t_{1}\right)}\right\rangle\left\langle q\left(t_{1}\right)\right\rangle\langle\dot{q}(0)\rangle
\end{aligned}
$$

where $\langle\ldots\rangle_{C}$ stands for the 3rd cumulant. The last three terms vanish since either $\langle\dot{q}(0)\rangle=0$ or $\left\langle q\left(t_{1}\right)\right\rangle=0$, the latter being true for any symmetric potential such as the harmonic oscillator (Sec.I C) or with no external potential in the MD simulations (Sec. III). With the equivalence of Eq. 4 and Eq. 7, the second term can be written as:

$$
\begin{aligned}
\left\langle\frac{\partial q\left(t_{1}+t_{2}\right)}{\partial p\left(t_{1}\right)}\right. & \rangle\left\langle q\left(t_{1}\right) \dot{q}(0)\right\rangle \\
= & \left\langle q\left(t_{2}+t_{1}\right) \dot{q}\left(t_{1}\right)\right\rangle\left\langle q\left(t_{1}\right) \dot{q}(0)\right\rangle \\
= & \left\langle q\left(t_{2}\right) \dot{q}(0)\right\rangle\left\langle q\left(t_{1}\right) \dot{q}(0)\right\rangle,
\end{aligned}
$$

This result can be compared directly to Eq. 18, which is correct in the limit of Gaussian dynamics of the velocity. It contains an additional term $\left\langle q\left(t_{1}+t_{2}\right) \dot{q}(0)\right\rangle$, which equals the first term of Eq. A10 in this limit. Hence, both expression would coincide when the correlation between time 0 and $t_{1}+t_{2}$ can be neglected. 
Alternatively, one may integrate Eq. 11 in parts, similar to Eq. A6:

$$
\begin{aligned}
& \left\langle\frac{\partial q\left(t_{1}+t_{2}\right)}{\partial p\left(t_{1}\right)} q\left(t_{1}\right) p(0)\right\rangle \\
& \quad=\left\langle q\left(t_{1}+t_{2}\right) q\left(t_{1}\right)\left(\frac{p\left(t_{1}\right) p(0)}{m k_{B} T}-\frac{\partial p(0)}{\partial p\left(t_{1}\right)}\right)\right\rangle
\end{aligned}
$$

If only the first term in the bracket is retained, Eq. 12 is obtained. The physical meaning of both terms in the bracket are very similar, which can be seen when isolating them as two-point correlation functions:

$$
\frac{1}{m k_{B} T}\left\langle p\left(t_{1}\right) p(0)\right\rangle=\left\langle\frac{\partial p(0)}{\partial p\left(t_{1}\right)}\right\rangle,
$$

in analogy to Eq. A6. In the limit $t_{1} \rightarrow 0$, the second term will be 1 always, $\partial p(0) / \partial p(0)=1$, while the first term will be 1 only in an averaged sense, $\left\langle p^{2}\right\rangle / m k_{B} T=1$. Hence, despite the equivalence in Eq. A13, the two terms don't cancel in Eq. A12.

Finally, it is illustrative to express the two terms of Eq. A12 for the limiting case of a harmonic oscillator with:

$$
q(t)=q(0) \cos (\omega t)+\frac{p(0)}{m \omega} \sin (\omega t)
$$

in which case one obtains together with Eq. A2 for

$$
\begin{aligned}
\frac{1}{m k_{B} T}\left\langleq \left( t_{1}\right.\right. & \left.\left.+t_{2}\right) q\left(t_{1}\right) p\left(t_{1}\right) p(0)\right\rangle \\
& =\frac{2 k_{B} T}{m^{2} \omega^{2}} \cos \left(\omega\left(t_{1}-t_{2}\right)\right),
\end{aligned}
$$

for

$$
\begin{aligned}
& \left\langle q\left(t_{1}+t_{2}\right) q\left(t_{1}\right) \frac{\partial p(0)}{\partial p\left(t_{1}\right)}\right\rangle \\
& =-\frac{k_{B} T}{m^{2} \omega^{2}}\left(\cos \left(\omega\left(t_{1}-t_{2}\right)\right)+\cos \left(\omega\left(t_{1}+t_{2}\right)\right)\right)
\end{aligned}
$$

and hence for Eq. A12

$$
\begin{aligned}
& \left\langle\frac{\partial q\left(t_{1}+t_{2}\right)}{\partial p\left(t_{1}\right)} q\left(t_{1}\right) p(0)\right\rangle \\
& \quad=\frac{k_{B} T}{m^{2} \omega^{2}}\left(\cos \left(\omega\left(t_{1}-t_{2}\right)\right)-\cos \left(\omega\left(t_{1}+t_{2}\right)\right)\right)
\end{aligned}
$$

In either case, the desired rephasing term, $\cos \left(\omega\left(t_{1}-t_{2}\right)\right)$, is present (the difference between Eq. 14 and Eq. A15 is explained when replacing $q\left(t_{1}+t_{2}\right)$ and $q\left(t_{1}\right)$ in the latter by their time-derivatives).
1 P. G. Debenedetti, J. Phys.- Condens Matter 15, R1669 (2003).

2 O. Mishima and H. E. Stanley, Nature 396, 329 (1998).

3 J. C. Palmer, F. Martelli, Y. Liu, R. Car, A. Z. Panagiotopoulos, and P. G. Debenedetti, Nature 510, 385 (2014).

4 P. Hamm and J. Savolainen, J. Chem. Phys. 136, 94516 (2012).

5 J. Savolainen, S. Ahmed, and P. Hamm, Proc. Natl. Acad. Sci. 110, 20402 (2013).

6 A. Shalit, S. Ahmed, J. Savolainen, and P. Hamm, Nat. Chem. 9, 273 (2017).

7 A. Berger, G. Ciardi, D. Sidler, P. Hamm, and A. Shalit, Proc Natl. Acad. Sci. USA 116, 2458 (2019).

8 E. Hahn, Phys. Rev. 80, 580 (1950).

9 A. Tokmakoff and M. D. Fayer, J. Chem. Phys. 103, 2810 (1995).

10 P. Hamm, M. H. Lim, and R. M. Hochstrasser, Phys. Rev. Lett. 81, 5326 (1998).

11 W. H. Hesselink and D. A. Wiersma, J. Chem. Phys. 73, 648 (1980).

12 T. Joo, Y. Jia, J.-Y. Yu, M. J. Lang, and G. R. Fleming, J. Phys. Chem. 104, 6089 (1996).

13 D. Sidler and P. Hamm, J. Chem. Phys. 150, 044202 (2019).

14 P. Hamm, J. Chem. Phys. 141, 184201 (2014).

15 V. Babin, C. Leforestier, and F. Paesani, J. Chem. Theory Comput. 9, 5395 (2013).

16 T. Hasegawa and Y. Tanimura, J. Chem. Phys. 125, 074512 (2006).

17 H. Ito, T. Hasegawa, and Y. Tanimura, J .Phys. Chem. Lett. 7, 4147 (2016).
18 G. S. Grest, S. R. Nagel, and A. Rahman, Solid State Commun. 36, 875 (1980).

19 O. M. Becker and M. Karplus, Phys. Rev. Lett. 70, 3514 (1993).

20 D. Xu, K. Schulten, O. M. Becker, and M. Karplus, J. Chem. Phys. 103, 3112 (1995).

21 D. Xu and K. Schulten, J. Chem. Phys. 103, 3124 (1995).

22 S. Saito and I. Ohmine, Phys. Rev. Lett. 88, 207401 (2002).

23 S. Mukamel, Principles of Nonlinear Optical Spectroscopy (Oxford University Press, Oxford, 1995).

24 M.-C. Bellissent-Funel and J. Teixeira, J. Mol. Struct. 250, 213 (1991).

25 Y. Tanimura and S. Mukamel, J. Chem. Phys. 99, 9496 (1993).

26 T. Steffen, J. T. Fourkas, and K. Duppen, J. Chem. Phys. 105, 7364 (1996).

27 T. Steffen and K. Duppen, Chem. Phys. 233, 267 (1998).

28 S. Saito and I. Ohmine, J. Chem. Phys. 108, 240 (1998).

${ }^{29}$ K. Okumura and Y. Tanimura, J. Chem. Phys. 107, 2267 (1997).

30 A. Ma and R. M. Stratt, Phys. Rev. Lett. 85, 1004 (2000).

31 T. l. C. Jansen, J. G. Snijders, and K. Duppen, J. Chem. Phys. 113, 307 (2000).

32 K. Okumura and Y. Tanimura, J. Phys. Chem. A 107, 8092 (2003).

33 S. Saito and I. Ohmine, J. Chem. Phys. 125, 84506 (2006).

34 Y. Nagata, T. Hasegawa, and Y. Tanimura, J. Chem. Phys. 124, 194504 (2006).

35 A. Tokmakoff, M. J. Lang, D. S. Larsen, G. R. Fleming, V. Chernyak, and S. Mukamel, Phys. Rev. Lett. 79, 2702 (1997).

36 D. A. Blank, L. J. Kaufman, and G. R. Fleming, J. Chem. 
Phys. 111, 3105 (1999).

37 D. A. Blank, L. J. Kaufman, and G. R. Fleming, J. Chem. Phys. 113, 771 (2000).

${ }^{38}$ L. J. Kaufman, J. Heo, L. D. Ziegler, and G. R. Fleming, Phys. Rev. Lett. 88, 207402 (2002).

39 O. Golonzka, N. Demirdöven, M. Khalil, and A. Tokmakoff, J. Chem. Phys. 113, 9893 (2000).

${ }^{40}$ K. J. Kubarych, C. J. Milne, and R. J. D. Miller, Int. Rev. Phys. Chem. 22, 497 (2003).

41 Y. L. Li, L. Huang, R. J. D. Miller, T. Hasegawa, and Y. Tanimura, J. Chem. Phys. 128, 234507 (2008).

42 P. Hamm and G. Stock, Phys. Rev. Lett. 109, 173201 (2012).

43 I. A. Finneran, R. Welsch, M. A. Allodi, T. F. Miller, and G. A. Blake, Proc. Natl. Acad. Sci. 113, 6857 (2016).

${ }^{44}$ I. A. Finneran, R. Welsch, M. A. Allodi, T. F. Miller, and G. A. Blake, J. Phys. Chem. Lett. 8, 4640 (2017).

45 M. Cho, J. Chem. Phys. 111, 4140 (1999).

${ }^{46}$ P. Hamm and A. Shalit, J. Chem. Phys. 146, 130901 (2017).

47 R. F. Loring and S. Mukamel, J. Chem. Phys. 83, 2116 (1985).

48 P. Hamm and M. T. Zanni, Concepts and Methods of 2D Infrared Spectroscopy (Cambridge University Press, Cambridge, 2011).

49 M. Khalil, N. Demirdöven, and A. Tokmakoff, Phys. Rev. Lett. 90, 47401 (2003).

50 S. Roy, M. S. Pshenichnikov, and T. L. Jansen, J. Phys. Chem. B 115, 5431 (2011).

51 N. G. Van Kampen, Stochastic Processes in Physics and Chemistry (Elsevier, Amsterdam, 1992).

${ }^{52}$ D. Van Der Spoel, E. Lindahl, B. Hess, G. Groenhof, A. E. Mark, and H. J. C. Berendsen, J. Comput. Chem. 26, 1701
(2005)

53 J. L. F. Abascal and C. Vega, J. Chem. Phys. 123, 234505 (2005).

54 T. Darden, D. York, and L. Pedersen, J. Chem. Phys. 98, 10089 (1993).

${ }^{55}$ G. Bussi, D. Donadio, and M. Parrinello, J. Chem. Phys. 126, 014101 (2007).

56 H. J. C. Berendsen, J. P. M. Postma, W. F. van Gunsteren, A. DiNola, and J. R. Haak, J Chem Phys 81, 3684 (1984).

57 M. J. Robertson, J. Tirado-Rives, and W. L. Jorgensen, J. Chem. Theory Comput. 11, 3499 (2015).

58 M. Sharma, R. Resta, and R. Car, Phys. Rev. Lett. 95, $187401(2005)$

59 H. Torii, J. Chem. Theory Comput. 10, 1219 (2014).

60 D. Sidler, M. Meuwly, and P. Hamm, J. Chem. Phys. 148, 244504 (2018).

61 D. C. Douglass, J. Chem. Phys. 35, 81 (1961).

62 J. B. Asbury, T. Steinel, K. Kwak, S. A. Corcelli, C. P. Lawrence, J. L. Skinner, and M. D. Fayer, J. Chem. Phys. 121, 12431 (2004).

63 S. Yeremenko, M. S. Pshenichnikov, and D. A. Wiersma, Chem. Phys. Lett. 369, 107 (2003).

64 M. L. Cowan, B. D. Bruner, N. Huse, J. R. Dwyer, B. Chugh, E. T. J. Nibbering, T. Elsaesser, and R. J. D. Miller, Nature 434, 199 (2005).

65 J. D. Eaves, J. J. Loparo, C. J. Fecko, S. T. Roberts, A. Tokmakoff, and P. L. Geissler, Proc. Natl. Acad. Sci. USA 102, 13019 (2005).

66 F. Perakis, S. Widmer, and P. Hamm, J. Chem. Phys. 134, 204505 (2011).

67 J. S. Kim, Z. Wu, A. R. Morrow, A. Yethiraj, and A. Yethiraj, J. Phys. Chem. B 116, 12007 (2012).

68 S. Habershon, D. E. Manolopoulos, T. E. Markland, and T. F. Miller, Annu. Rev. Phys. Chem. 64, 387 (2013). 